\title{
Impact Dynamics of a Flexible Beam with Rotation Yue-Chen DUAN ${ }^{1, a}$, Ting-Ting WANG ${ }^{1}$ and Wei-Wei ZHANG ${ }^{1}$
}

\author{
${ }^{1}$ School of Mechanical Engineering, Zhengzhou University, No. 100 of Science Road, Zhengzhou, \\ China 450001
}

a Corresponding author: duanyuechen@tom.com

Keywords: Flexible beam, Impact, Dynamics, Continuous contact force model.

\begin{abstract}
Based on the continuous contact force model, the rigid-flexible coupling impact dynamics of a radial rotating flexible beam is investigated. The transversal deformation and nonlinear coupled deformation, which means the longitudinal shortening caused by transversal deformation, is considered. According to the rigid-flexible coupling dynamic theory of flexible multibody system, the continuous dynamic equations of the system are established by using the recursive Lagrange method. The impact force is calculated by a nonlinear spring-damper model based on Hertz contact theory and nonlinear damping theory. By introducing the concept of impact potential energy, the system's rigid-flexible coupling impact dynamic equations are obtained. An impact dynamic simulation example is given to validate the impact model and the dynamic method, and the dynamic responses such as the impact force, the angular velocity and the flexible deformation in the whole procedure are got. The large overall motion, the small deformation motion and the impact effect are coupled.
\end{abstract}

\section{Introduction}

In the engineering fields, such as aerospace, robotics, and vehicles, the recent mechanical systems are with more complex configurations, lighter materials, larger scales, higher speed, and more accurate movements. And the accurate methods to describe the dynamics of flexible multibody systems with large overall motion becomes much more important and difficult. Rigid-flexible coupling problem is one of the hot subjects in current theoretical and applied mechanics. The development of the flexible multibody system dynamics can be summarized as several stages: the kinematical-elastic dynamics (KED method), the zeroth-order rigid-flexible coupling dynamics, and some higher level rigid-flexible coupling dynamics (including the study on dynamic stiffening, and the first-order rigid-flexible coupling dynamics, etc.). Researchers are still doing explorations in mechanical theory of rigid-flexible coupling dynamics, general dynamic modeling method, stable and efficient numerical algorithms [1-2].

As the complexity of the work environment, higher requirements of performance and existence of clearance between components, flexible multibody system is likely to impact with the surrounding environment or itself in operation. And impact will make great effects on the dynamics of flexible multibody system. It will stimulate higher order modes of flexible bodies, and affect the system's operating stability and accuracy [3-4]. Considering the impact, rigid-flexible coupling dynamics of flexible multi-body system becomes more complex, as well as the dynamic modeling and numerical solution. The key to solve the impact dynamics of flexible multibody system is an accurate rigidflexible coupling dynamic model of the system, which can make correct treatment of the impact process, and find a reasonable and stable calculating method.

According to the different assumptions on the impact process, the impact model can be divided into three types: 1) the impulse-momentum method, based on the generalized impulse-momentum equations and the coefficient of restitution equations; 2) the continuous contact force method, based on the quasi-static contact theory; 3) the contact constraint method, based on the kinematic constraint conditions of the impact process. There are a lot of impact models which have been used on the impact dynamics of multibody system by far [5-6]. For the continuous contact force method, the impact process is no longer considered as an instantaneous process, so the time history of the impact force can be studied. Besides, the dimensions of the dynamic equations are constant, and do 
not change with contact status. Therefore, the continuous contact force model has been widely used. Scholars have proposed a lot of continuous contact force models, such as the Hertz contact model, the linear spring-damper model, the nonlinear spring-damper model and the elastic-plastic contact model [7-10], which have been used on the impact dynamics of flexible multibody systems.

The research on the impact dynamics of flexible bodies with overall motion is relatively weak, which is essentially a temporal and spatial multi-scale, strongly nonlinear, highly coupled and noncontinuous dynamic problem. It presents us with new challenges and research hotspot. In this paper, the dynamics of a radial rotating flexible beam with large overall motion, which impacts with a fixed surface, is studied. Based on the geometric nonlinearity, the axial shortening caused by transverse deformation is considered here. The assumed mode method is used to describe the deformation. Then a rigid-flexible coupling dynamic model considered the nonlinear geometric stiffening effect is created by using Lagrange equations. The impact force is calculated by using the nonlinear spring-damper model. And the concept of impact potential energy is introduced, so that the generalized impact forces will be programmably put into the system's dynamic equation. Then the system's rigid-flexible coupling impact dynamic model is established. And the judgment law of contact-impact is established at the same time. The impact dynamic simulation example is given to validate the impact dynamic method.

\section{System's Description}

The flexible beam with large overall rotating is shown in Figure 1. The flexible beam is an EulerBernoulli beam, and the deformation is small. The parameters of the flexible beam are given as the length L, the Young's modulus E, the area moment of inertia I, the cross-sectional area A, the mass density $\rho$. An inertial coordinate system OXY is fixed at the rotating axis $\mathrm{O}$ of the flexible beam, and a floating coordinate system Oxy is fixed at the flexible beam where $\mathrm{x}$-axis is coincident with the neutral axis of the beam in its undeformed condition.

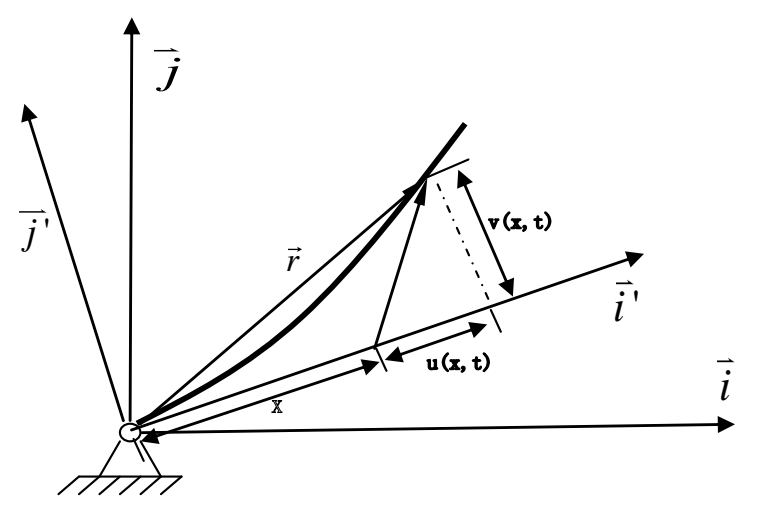

Figure 1. Dynamic model of the flexible beam.

Since the beam is a slender beam, the deformation of the axial tension and compression is ignored. The transverse deformation $\mathrm{v}(\mathrm{x}, \mathrm{t})$ and the axial shortening $\mathrm{ux}(\mathrm{x}, \mathrm{t})$ caused by transverse deformation, which is called the coupling deformation, are considered.

The axial shortening caused by transverse deformation can be got by Equation (1).

$$
u_{x}(x, t)=-\frac{1}{2} \int_{0}^{x}\left[\frac{\partial v(\xi, t)}{\partial \xi}\right]^{2} \mathrm{~d} \xi
$$

Since this coupling deformation ux is displayed as an inertial force item in the final dynamic equation, it will bring essential influence to the system's dynamics when the overall motion of the flexible body is in high speed. The coupling dynamic model of the system resulted from this kind of deformation field considers geometric nonlinear stiffening effect. 


\section{Impact Model}

A nonlinear spring-damper model is used to describe the contact-impact process. The elastic contact force based on the contact theory is used to reflect the elastic restoring force, and the nonlinear damping force is used to reflect the energy loss during the impact process, as shown in Figure 2. The elastic contact force is in the opposite direction with the embedding direction of the two impacting bodies, and the nonlinear damping force is in the opposite direction with the direction of the relative velocity.

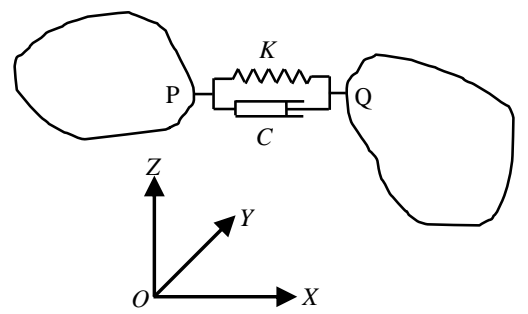

Figure 2. Nonlinear spring-damper model.

Based on the Hertz contact theory and the nonlinear damping theory, the impact force $\mathrm{F}$ of the nonlinear spring-damper model is expressed as follows.

$$
F=\left(F_{k}+F_{d}\right) \cdot L(\gamma)
$$

The contact force $F_{k}$ according to contact theory reflects the elastic restoring force in the impact process, and the nonlinear damping force $F_{d}$ reflects the energy loss during impact. $\gamma$ is the indention. And $L(\gamma)$ is the logic function to determine contact, based on the embedding amount $\gamma$ between the impacting bodies. The contact force is got by Equation (3).

$$
F_{k}=K \gamma^{3 / 2}
$$

In which, $K$ is the contact stiffness which is related to the contact geometry and material properties, and can be obtained by experiment.

The damping force is got by Equation (4).

$$
F_{d}=C \gamma \dot{\gamma}
$$

Where $C$ is the damping coefficient.

\section{Impact Dynamic Equations}

As shown in Figure 1, in the floating coordinate system, after the beam deforms, the position vector of a point $P(\mathrm{x}, 0)$ in the beam when undeformed can be expressed as follow.

$$
\vec{r}=\left(x+u_{x}\right) \vec{i}+v \vec{j}^{\prime}
$$

The velocity of the point is:

$$
\dot{\vec{r}}=\left(\dot{u}_{x}-v \dot{\theta}\right) \vec{i}^{\prime}+\left[\left(x+u_{x}\right) \dot{\theta}+\dot{v}\right] \vec{j}^{\prime}
$$

The total kinetic energy $T$ of the system is:

$$
T=\frac{1}{2} \int_{0}^{L} \rho A(\dot{\vec{r}} \cdot \dot{\vec{r}}) \mathrm{d} x
$$

The system's potential energy includes the elastic potential energy of the flexible beam, the gravitational potential energy, and the impact force potential energy generated by the impact force which is treated as potential force. And the impacting potential energy is introduced to conveniently calculate the generalized impact forces. The total potential energy $V$ of the system is: 


$$
V=V_{L}+V_{g}+V_{F}
$$

In which, $V_{L}$ is the elastic potential energy of flexible beam, and $V_{g}$ is the gravitational potential energy.

$$
\begin{gathered}
V_{L}=\frac{1}{2} \int_{0}^{L} E I\left(\frac{\partial^{2} v}{\partial x^{2}}\right)^{2} \mathrm{~d} x \\
V_{g}=\int_{0}^{L}-\left(\rho A g^{\mathrm{T}} W h\right) \mathrm{d} x=-g^{\mathrm{T}} W R
\end{gathered}
$$

In which, $g$ is the gravity vector expressed in the inertial coordinate system, and $h$ is the microelement's position vector in the floating coordinate system.

In each integration step, the impact force is treated as potential force. The impact force potential energy is:

$$
V_{F}=-n_{F}^{T} F W h_{F}
$$

In Equation (12), $n_{F}$ is the unit vector which denotes the direction of the impact force, and expressed in the inertial system. $F$ is the magnitude of the impact force. $W$ is the homogeneous transformation matrix from the inertial system to the floating system. And $h_{F}$ is impact point's position in the floating system. In the impact process the impact force potential energy exists, and remains zero in other time.

Assumed modes method is used to describe the transverse deformation of the flexible beam.

$$
v(x, t)=\sum_{i=1}^{N} \varphi_{y i}(x) B_{i}(t)
$$

In which, $\varphi_{y i}(\mathrm{x})$ are the beam's transverse modal functions, $B_{i}(t)$ are the modal coordinates, and $N$ is the mode truncation number.

The nonlinear coupling deformation in Equation (1) can be expressed as follow.

$$
u_{x}(x, t)=-\frac{1}{2} \sum_{i=1}^{N} \sum_{j=1}^{N} e_{i j}(x) B_{i}(t) B_{j}(t)
$$

Where

$$
e_{i j}(x)=\int_{o}^{x} \varphi_{y i}^{\prime}(\xi) \cdot \varphi_{y j}^{\prime}(\xi) \mathrm{d} \xi
$$

The generalized coordinates are taken as $z=\left[\theta, B_{1}, B_{2}, \cdots B_{N}\right]^{\mathrm{T}}$. By Using the Lagrange equations, and put the substitution of the kinetic energy and the potential energy expressions into them, one can get the global rigid-flexible coupling impact dynamic equations after complex derivation.

$$
J \ddot{z}=Q
$$

Where $\boldsymbol{J}$ is the generalized mass matrix, and $\boldsymbol{Q}$ is the generalized force vector.

$$
\begin{gathered}
\boldsymbol{J}=\left[\begin{array}{cccc}
J_{0} & P_{1} & \cdots & P_{N} \\
M_{1} & M_{11} & \cdots & M_{1 N} \\
\vdots & \vdots & \ddots & \vdots \\
M_{N} & M_{N 1} & \cdots & M_{N N}
\end{array}\right] \\
\boldsymbol{Q}=\boldsymbol{Q}^{\mathrm{I}}+\boldsymbol{Q}^{\mathrm{II}}
\end{gathered}
$$

Where $Q^{\mathrm{I}}$ is the generalized force vector without impact. And $Q^{\mathrm{II}}$ is the impact generalized force vector, and will be zero when impact does not occurs. 
As the coupling deformation is considered in dynamic modeling, the dynamic equations have additional coupling items. These additional coupling items appear in the generalized mass matrix and the generalized force vector, and the traditional dynamic model does not contain the items. When the coupling items are ignored, the lower right matrix $\left[M_{i j}\right]$ is a constant matrix, as is the mass matrix in structural dynamics. When adding the coupling items, the mass matrix is no longer a constant matrix, but a nonlinear and inertial highly coupled matrix related to the generalized coordinates.

\section{Simulation}

An impact dynamic simulation example of a flexible beam with rotation as shown in Figure 1 is given. The structural parameters of the system are as follows: $\rho=2.767 \times 10^{3} \mathrm{~kg} / \mathrm{m}^{3}, E=68.95 \mathrm{GPa}$, $L=1.0 \mathrm{~m}, I=7.85 \times 10^{-9} \mathrm{~m}^{4}, A=3.14 \times 10^{-4} \mathrm{~m}^{2}$. The flexible beam is given an initial angular velocity. And the beam tip impacts with ground at the initial moment. In order to fully consider the effect of higher-order modes on impact dynamics, the mode truncation number is set to 6 .
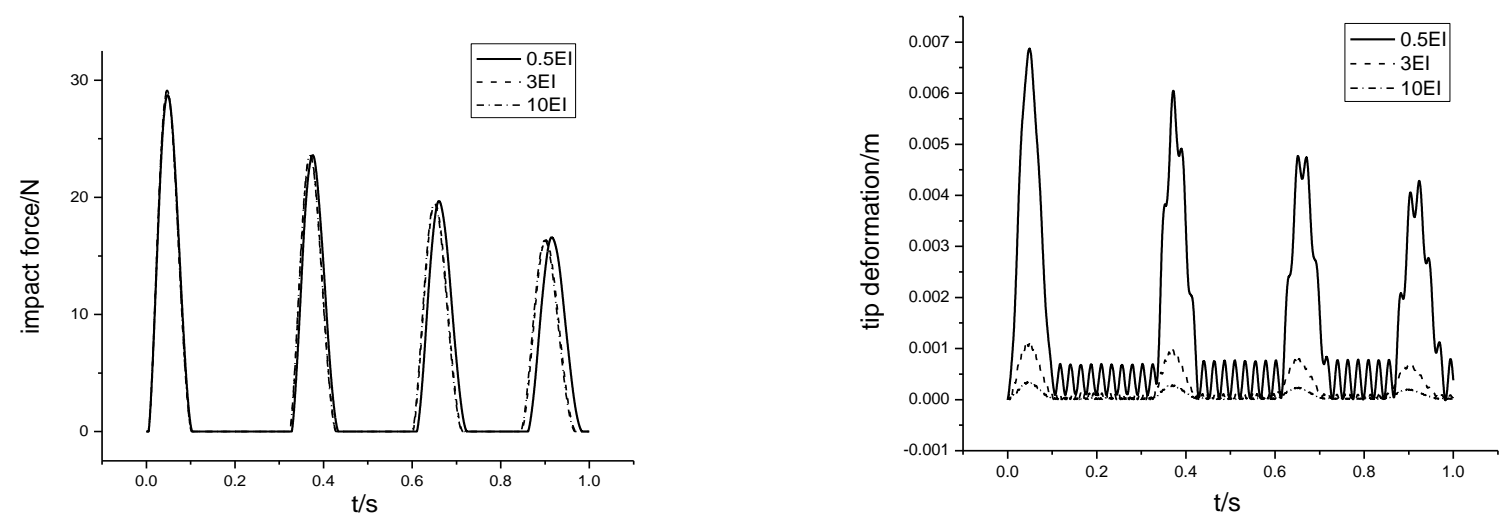

Figure 3. Impact force.

Figure 4. Tip transverse deformation of the beam.

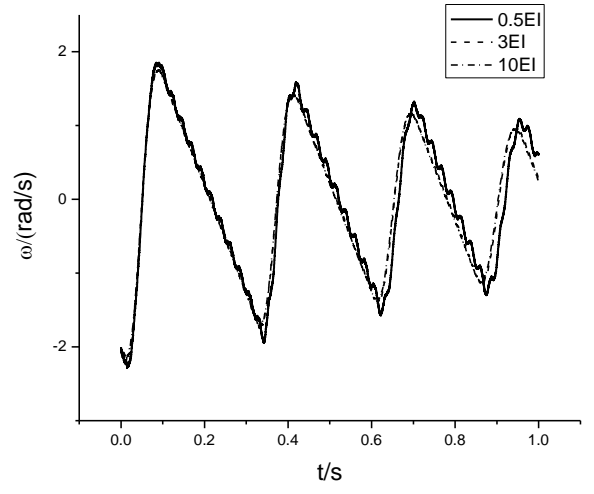

Figure 5. Angular velocity.

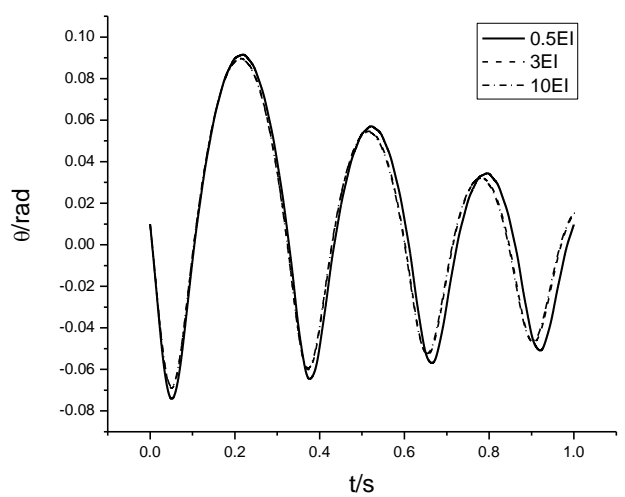

Figure 6. Angular displacement.

Several results with different flexibility of the beam are given in Figures 3-6. Time history curves of impact force are shown in Figure 3. It can be seen that the impact occurs for 4 times. Because of the energy loss caused by impact, the amplitude of the impact force becomes smaller and smaller in later impacts. With the flexible beam stiffness increasing, the time between two impacts has been reduced, and the amplitude of the impact force decreases slightly, but overall the difference was small. This is because the beam's deformation decreases as the stiffness increases, resulting in decreasing of the amount and time of reduction. So the impact process ends faster and the impact force becomes less. The tip transverse deformation of the flexible beam is shown in Figure 4. The transverse deformations have been significant different as the beam's stiffness 
increases. When the impact occurs, the deformation increases obviously. As the beam's bending stiffness increases, the deformation amplitude dramatically reduced during both impact and nonimpact process. The changes of overall angular displacement and velocity of the flexible beam are shown in Figures 5-6. The variation discipline of the angular displacement and velocity is similar to that of the impact force. It also reflects that the magnitude of the overall motion is slightly reduced as the beam's stiffness increases.

\section{Conclusions}

A flexible beam with large overall rotation impacting with a fixed surface is studied in this paper. Based on rigid-flexible coupling multi-body system dynamic theory, the system's coupling dynamic equations are established. The nonlinear spring-damper model based on Hertz contact theory is used to describe the impact process. The system's impact dynamic model is obtained. And the judgment law of contact-impact is established. It lays a theoretical foundation to solve the impact problems of flexible multibody system dynamics. Simulation results verify the reasonability of the method, and describe the whole impact process. The effects of flexibility on system's impact dynamics are compared.

\section{Acknowledgments}

This work is financially supported by the National Natural Science Foundation of China (11402234) and the Key Scientific Research Foundation of Henan Province, China (16A130005, 14A460003).

\section{References}

1. D. G. Zhang. Applied Mathematics and Mechanics (English Edition), 1283, 30 (2009)

2. W. Schiehlen. Multibody System Dynamics, 3, 18 (2007)

3. Y. C. Duan. Applied Mathematics and Mechanics (English Edition), 1393, 34 (2013)

4. A. A. Shabana. Multibody System Dynamics, 189, 1 (1997)

5. S. K. Dwivedy, P. Eberhard. Mechanism and Machine Theory, 749, 41 (2006)

6. D. M. Flickinger, A. Bowling. Multibody System Dynamics, 249, 23 (2010)

7. Y. C. Duan, D. G. Zhang. Advances in Mechanical Engineering, 1, 2013 (2013)

8. H. M. Lankarani, P. E. Nikavesh. Journal of Mechanical Design, 369, 112 (1990)

9. M. Payr, C. Glocker. Nonlinear Dynamics, 361, 41 (2005)

10. A. S. Yigit. Journal of Dynamic Systems, Measurement, and Control, 527, 117 (1995) 